\title{
Fasting versus non-fasting Lipid profile in the clinical practice
}

\author{
Umakanth M \\ Faculty of Health care sciences, Eastern university-Sri Lanka
}

\section{Abstract}

There are pros and cons doing fasting and random non-fasting lipid profile test. However, when we consider cardiovascular risk, non-fasting lipid test more reliable than fasting test. In current practice, using fasting lipid profile was challenged in 2007 by two studies that showed that random non-fasting triglyceride (TG) could be superior than fasting TG in predicting risk of cardiovascular risk. Postprandial concentrations of triglyceride were higher than fasting. However, levels of non-fasting triglycerides are better at predicting future cardiovascular events than levels of fasting triglycerides. Random non-fasting lipid test is not a new phenomenon, it already has been practicing in Denmark since 2009. Moreover, NICE guidelines have recommended non-fasting test in the primary prevention setting since 2014. As the major shift in newer guidelines reflects the changing focus of risk assessment from LDL to non-HDL cholesterol (apolipoprotein B) as a better predictor of cardiovascular risk, indirectly tell us non-fasting lipid level superior than fasting lipid. This is a review of the published literature. The databases Medline, and Google Scholar were searched using the terms "fasting lipid profile, non-fasting lipid profile, and fasting versus non-fasting lipid profile. Database were merged and a duplicate was removed. The aim of the review is to compare pros and cons of both fasting and non-fasting measurements in our clinical practice.

Keywords: Lipids, non-fasting lipid profile, fasting lipid profile and lipoprotein

Copyright: This is an open-access article distributed under the terms of the Creative Commons Attribution License, which permits unrestricted use, distribution, and reproduction in any medium, provided the original author and source are credited (CC BY 4.0)

Received: 24th June 2018 Accepted with revision: 24th July 2018 Published: 31 st Aug 2018

Correspondence: email: mumakanth1972@gmail.com

https://orcid.org/0000-0002-0255-6657

This is an open-access article distributed under the terms of the Creative Commons Attribution License, which permits unrestricted use, distribution, and reproduction in any medium, provided the original author and source are credited (CC BY 4.0) 


\section{Introduction}

Lipid profile is measured for cardiovascular risk forecast and has now become virtually a routine test (1). The test includes four basic parameters such as total cholesterol (TC), high -density lipoproteins (HDL), low- density lipoproteins (LDL) and triglycerides (TG). It is usually done in fasting blood specimen. A fasting lipid profile test is one that is performed after a definite period of time during which a person has not eaten or drunk anything with the exception of pure water. A non-fasting cholesterol test, sometimes referred to as a random cholesterol test, measures cholesterol level without any restrictions on dietary intake. It is understandable that random non-fasting lipid measurements may even better than fasting lipid test (2).

In Denmark, non-fasting lipid testing has been used since 2009, while guidelines from the National Institute for Health and Care Excellence in the United Kingdom also support non-fasting lipid testing in the primary prevention setting $(3,4)$. Some cholesterol tests require in a fasting state, while others are not affected by food intake. According to the Health Services at Columbia, fat and cholesterol components are usually only measurable for approximately 10 hours after a meal. Because of this, most physicians recommend fasting for a period of at least 12 hours before a fasting cholesterol test. Alcohol can increase the triglyceride levels significantly, so abstinence from alcohol for at least 24 hours before a fasting cholesterol test is required.

However, Standard practice in most countries today calls for patients to fast for at least 8 hours prior to having blood drawn. Though, in some patients lengthy fasting may not be practicable or the patient may present having not fasted. In these situations, the test report should indicate clearly that the patient has not fasted. If the testing returns abnormal results, then it is recommended that the patient should be further assessed by collecting a fasting specimen. The non-fasting concentration of TC, HDL, LDL, apolipoprotein A1 and apolipoprotein B were lower than fasting concentration. These minimal changes of lipid fraction will not make any consequences in the clinical practice $(5,6)$.

\section{Current practice}

Postprandial concentrations of triglyceride were higher than fasting. However, levels of non-fasting triglycerides are better at predicting future cardiovascular events than levels of fasting triglycerides. In practice, using fasting lipid profile was challenged in 2007 by two studies that showed that random non-fasting TG could be superior than fasting TG in predicting risk of cardiovascular risk (7). A wellknown study including 26509 women found that increased concentrations of TG in both fasting or non-fasting state connected with increased cardiovascular risk (8). Moreover, Copenhagen City Heart Study revealed that gradually higher concentrations of random non-fasting TG were associated with increasingly higher risk of ischemic heart disease, myocardial infarction, and all-cause mortality (6).

Lipid profile should be measured in all adults over 45 years of age as part of valuation of overall Cerebrovascular disease (CVD) risk. The outcome of testing will be low in those less than 45 years of age and this should be held in reserve for those who are at increased risk because of their personal or family history, or the presence of other disease such as diabetes mellitus or chronic kidney disease. In addition to that if a patient is started on lipid-lowering medication their serum lipids should be measured at sixmonth intervals to check that they are reaching their wanted values. Non-fasting cholesterol levels (TC, non-HDL-C, and LDL-C) are comparable to fasting cholesterol levels in predicting ASCVD, however non-fasting TG levels showed even stronger associations with ASCVD compared with fasting results $(7,9)$.

At present, doing serum TC and serum TG in a fasting specimen are the simplest and cheapest lipid measurements. However, the quantity of HDL-C and LDL-C, as markers of the anti and pro-atherogenic lipid particles, which gives more information and should be requested routinely. The reason for this is that some people with mildly increased TC may actually be at lower risk because their HDL-C is high, and their LDL-C is relatively low. People with low serum TC generally have low LDL-C, but they may also have low HDL-C concentrations. However, other tests such as lipid electrophoresis and apolipoprotein measurement should be reserved for those with unusual lipid disorders. However, people taking lipid-lowering therapy should be observed with LDL-C, HDL-C and TG values. Liver function tests and creatine kinase $(\mathrm{CK})$ should be measured at baseline and repeated during therapy only if adverse reactions to medication are suspected. Although recent reviews are encouraging about the safety of long-term lipid-lowering therapy, treatment is generally interrupted if Alanine transaminase (ALT) exceeds 2 to 3 times the upper limit of normal, or if unexplained creatine kinase $(\mathrm{CK})$ rise exceeds 5 to 10 times the upper limit of normal (10).

\section{Fasting versus non-fasting lipid test}

In 2013, according to the guidelines released by the American College of Cardiology and the American Heart Association cited that non-fasting lipid tests can be used for assessing cardiovascular risk, but still recommended a fasting lipid panel prior to statin initiation $(11,12)$. However, the European panels endorsed that non-fasting blood samples be "routinely used for the assessment of 
plasma lipid profiles, with laboratory reports flagging abnormal values on the basis of desirable concentration cut-points. Non-fasting and fasting measurements should be complementary but not mutually exclusive (11). Furthermore, the panel transcribed that non-fasting testing is generally appropriate in patients undergoing an initial lipid profile or cardiovascular risk assessment, those who are admitted with acute coronary syndrome, and for children, the elderly, and patients with diabetes. It is also appropriate if the patient prefers it. However, in certain conditions in which a fasting lipid profile may be preferred such as non-fasting triglycerides $5 \mathrm{mmol} / \mathrm{L}(440 \mathrm{mg} / \mathrm{dL})$, known hypertriglyceridemia followed in lipid clinic, recovering from hyper triglyceridemic pancreatitis, starting medications that cause severe hypertriglyceridemia (13). Former guidelines for assessing cardiovascular risk in patients recommend that lipid profiles are measured with the patient in a fasting state for at least 8 hours to decrease within individual variability in results. In addition, the majority of prospective studies that have been used to create modern cardiovascular risk prediction strategies and also clinical trials of statin treatment, have incorporated fasting lipid profiles. It is understandable that comparing with fasting and non-fasting measurements, TC, HDL, and LDL cholesterol measurements are slightly high in fasting state than non-fasting state, in contrast TG measurements are high in non-fasting state (5). These minor and transient changes in lipid concentrations appear to be clinically insignificant. Further, Langsted et al cited that reduction in total and LDL cholesterol at 1-3 $\mathrm{h}$ after the last meal detected in individuals with and without diabetes became statistically insignificant $(4,14)$.

\section{Pathogenesis of lipid metabolism}

Lipids are insoluble in aqueous solution and are transported in plasma in association with proteins(apolipoproteins) in the form of lipoproteins. The lipoproteins solubilize the lipids and provide an efficient transport system for them. If the system fails, the plasma lipids concentration will increase. In the long-term high plasma cholesterol level is associated with an increased risk of atherosclerosis. There are five main classes of lipoproteins, chylomicron (CM), very-low-density lipoproteins (VLDL), low- density lipoproteins (LDL), high- density lipoproteins (HDL). They are classified according to increased density. Dietary fat and cholesterol absorbed by the duodenum and proximal jejunum are used to produce chylomicrons that are secreted at the lateral borders of enterocytes and enter mesenteric lymphatics. They access the plasma via the thoracic duct and are rapidly metabolized by lipoprotein lipase (LPL) to yield chylomicron remnants. These are taken up by remnant receptors (LRP1/HSPG) and by LDL receptors in the liver. Free fatty acids liberated by the action of LPL are available to adipose tissue for storage and to other tissues for use as energy substrates (15). Lipid derived from remnants and from lipolysis of adipose tissue is reassembled in the liver as VLDL particles, which are secreted into the plasma. Abnormal lipid metabolism in insulin resistance is mediated in large part by overproduction of VLDL, an event that occurs through disruption of signaling downstream of the insulin receptor and the insulin receptor substrate (IRS) adapter proteins. VLDL particles are metabolized by LPL to yield IDL particles, which are metabolized by LPL and hepatic lipase to yield LDL particles. Thus, LDL is derived from VLDL, which helps explain why treatment to lower triglycerides (carried by VLDL) is frequently associated with at least transient increases in LDL. IDL can be taken up by the liver through an apo-E-dependent process, and LDL is taken up by the liver through the binding of apoB100 to LDL receptors. Thus, the measurement of lipids in the fasting or non-fasting state does not significantly affect the aggregate apolipoprotein B concentration under most circumstances (16). Small VLDL particles, IDL particles, and LDL particles may be taken up by peripheral tissues to deliver nutrients, cholesterol, and fat-soluble vitamins. When present in excess, each of these lipoproteins may be atherogenic (15).

\section{Component of lipid profile}

Lipid profile measurements consists of, TC, LDL cholesterol, HDL-cholesterol, and TG. However, TC, HDL cholesterol, and triglycerides are measured directly, while LDL cholesterol usually measured indirectly by the Friedewald equation (LDL=TC-HDL-TG/5) if triglycerides are less than $400 \mathrm{mg} / \mathrm{dl}(12,17)$. It often has been expected that the calculation of LDL cholesterol using the Friedewald equation requires a fasting lipid profile; however, there is now evidence suggesting that this is not necessarily even non-fasting lipid test can entertain (18). Certainly, several studies have established that lipids and lipoproteins, including calculated LDL cholesterol, display only minimal and clinically insignificant changes in response to food intake (9).

LDL-cholesterol also can be measured by direct method, if TG level are above $400 \mathrm{mg} / \mathrm{dl}$. The standard lipid profile consists of lipids, lipoproteins and apolipoproteins. Calculated remnant cholesterol is non-fasting total cholesterol minus low-density lipoprotein cholesterol minus high- density lipoprotein cholesterol (Remnant cholesterol=TC-LDL-HDL). Calculated non-high-density lipoprotein cholesterol is total cholesterol minus highdensity lipoprotein cholesterol (Non-HDL=TC-HDL) (19). Lastly, apolipoprotein B and apolipoprotein A1 can be used as alternatives to non-high-density lipoprotein and high-density lipoprotein cholesterol, but these measurements come at an extra cost. Interestingly, major shift in newer guidelines reflects the changing focus of risk assessment from LDL to non-HDL cholesterol (apolipoprotein B) as a better predictor of risk $(20,21)$. 


\section{Rationale behind the non-fasting lipid profile}

It is obvious that people eat several meals during the day, it could be three times a day or with snacks in between the main meal. This indicates that postprandial state is prevails than fasting state. In practice, the lipid profile measurement is measures in the fasting state at least 12-14 hours. Hence, it may not reflect the daily average plasma lipid and lipoprotein concentrations (22). Secondly, available literature cited that there are no advantages doing fasting over non-fasting lipid measurements. The main advantage of performing non-fasting is simple, convenient to the vulnerable group especially, elderly, diabetes, and patient with multiple comorbidities (23). Furthermore, it is samples and more convenient for the patient and the physician (Table 1) (24). Thirdly, random non-fasting reduces the burdened by large volume of early morning sample. Doing random lipid test is not a new phenomenon, it has been followed up by Denmark since 2009. They can be used to derive non-HDL-C, a measure that is superior to LDL-C for risk assessment as it includes atherogenic TG- rich lipoproteins. In the past decade, several essential studies (including the Women's Health Study, the Copenhagen City Heart Study, and the Copenhagen General Population Study) found that non-fasting TG were at least as good, if not better than fasting TG in predicting future risk of cardiovascular events (25).

\section{Rationale behind the fasting lipid profile}

Fasting lipid profile measurement is not a new one, it has been practicing for over the half century. Secondly, the existing lipid profile reference values were established on fasting state (26). It is superior for assessment of severe hypertriglyceridemia as they reduce postprandial variation. Calculation of LDL cholesterol by Friedewald equation also depends on the TG level. Small increment in TG level significantly affect the LDL-cholesterol as well. On the other hand, a fasting sample helps greatly in the diagnosis of genetic dyslipidemia and helps determine response to therapy of those with hyperlipidemic pancreatitis (Table 1). However, a fasting sample is ideal if cardiovascular disease (CVD) risk assessment is based on total cholesterol, LDL cholesterol or non-HDL cholesterol but HDL cholesterol, triglycerides, total/HDL cholesterol ratio and apolipoprotein A predict CVD when measured non- fasting (9). Furthermore, TG level increase with meal, this increment varies with time with highest level reached 5 hours after lunch (27). Further, the cut off levels of random non-fasting TG for cardiovascular risk have not yet been defined. Finally, most of the randomized lipid-lowering trials have used fasting lipid measurements and, in order to follow evidence-based practice, fasting blood sampling has often been the standard in day to day practice.

\section{Table 1: Suggested indications for fasting and non-fasting lipid profile test}

\section{Fasting}

Non-fasting triglycerides $5 \mathrm{mmol} / \mathrm{L}(440 \mathrm{mg} / \mathrm{dL})$

Known hypertriglyceridemia followed in lipid clinic

Recovering from hypertriglyceridemic pancreatitis

Starting medications that cause severe

hypertriglyceridemia

\section{Non-fasting}

Initial lipid profile testing in any patient

For cardiovascular risk assessment

Patients admitted with acute coronary syndrome

In children

If preferred by the patient

In diabetic patients

Patients on stable drug therapy

In the elderly
It is understandable that following recommendations are postulated in favor of fasting and non-fasting lipid test 
which based on the available literature. The National Clinical Guideline Center (NICE) and Joint British Societies recommended in 2014, that a fasting sample is not needed for routine clinical care. In addition to that most recently, in 2016, the European Atherosclerosis Society and the European Federation of Laboratory Medicine recommended using non-fasting lipid testing for routine clinical practice and provided specific cut-points for desirable fasting and non- fasting lipid levels (28). This major shift in newer guidelines reflects the changing focus of risk assessment from LDL to non-HDL cholesterol (apolipoprotein B) as a better predictor of risk.

Current guidelines cited that, LDL-cholesterol values may not be needed for some clinical scenarios. For several risk estimators, LDL is not included, but rather total cholesterol and high-density lipoprotein (HDL) cholesterol, both of which vary little between the fasting and non-fasting state (29). Therefore, in the estimation of initial risk among the primary prevention patients who are not on lipid-lowering therapy, random non-fasting lipid measurements would be acceptable (30). In addition to that, the screening and follow-up of patients with a family history of genetic (familial) hyperlipidemia and/or premature atherosclerotic cardiovascular disease (ASCVD) fasting sample is recommended. An LDL cholesterol $>190 \mathrm{mg} / \mathrm{dl}$ is the most common result suggestive of familial hyperlipidemia, where a fasting measure is recommended. Preferably, measurement of lipids such as triglycerides (a criterion of the metabolic syndrome) is recommended to be measured in the fasting state. However, non-fasting measures including TG $>200 \mathrm{mg} / \mathrm{dl}$ and a low HDL $(40 \mathrm{mg} / \mathrm{dl}$ in men or $<50 \mathrm{mg} / \mathrm{dl}$ in women), in the setting of a hemoglobin A1c $>5.6 \%$ would be consistent with traditional metabolic criteria and thus allow for therapeutic interventions to begin soon after results are measured. Thus, the authors of the commentary recommend that for the assessment of metabolic syndrome, non-fasting would be acceptable. In a recent statement on hypertriglyceridemia, the American Heart Association suggested that providers could use non-fasting triglycerides $>200 \mathrm{mg} / \mathrm{dl}$ to identify hyper triglyceridemic states. In most cases, when an elevated result is observed, then a repeat fasting triglyceride can be measured in 2 to 4 weeks. In the cases when extreme levels are observed, for example approximately $1000 \mathrm{mg} / \mathrm{dl}$, there is no need for repeat of fasting lipids prior to treatment.

\section{Conclusion}

Interestingly, available evidence is lacking that fasting is superior to random non-fasting when evaluating cardiovascular risk assessment. As the major shift in newer guidelines reflects the changing focus of risk assessment from LDL to non-HDL cholesterol (apolipoprotein B) as a better predictor of cardiovascular risk, as a result of doing random non-fasting lipid measurements superior than fasting state. However, we need to aware of other indications for fasting lipid profile test such as non-fasting triglycerides $0.5 \mathrm{mmol} / \mathrm{L} \quad(440 \mathrm{mg} / \mathrm{dL})$, known hypertriglyceridemia followed in lipid clinic, recovering from hyper triglyceridemic pancreatitis, starting medications that cause severe hypertriglyceridemia and require additional laboratory tests that entail fasting or morning samples, such as fasting glucose, and therapeutic drug monitoring.

\section{References}

1. Nayor M, Vasan RS. Recent update to the us cholesterol treatment guidelines: A comparison with international guidelines. Circulation. 2016;133(18):1795-806.

2. Nordestgaard EBG, Wierzbicki AS, Vesper H, Mora S, Stone NJ, Genest J, et al. Q \& A Nonfasting Sample for the Determination of Routine Lipid Profile : Is It an Idea Whose Time Has Come? Q \& A. 2016;435.

3. Nordestgaard BG, Langsted A, Mora S, Kolovou G, Baum H, Bruckert E, et al. Fasting is not routinely required for determination of a lipid profile: Clinical and Laboratory implications including flagging at desirable concentration cutpoints-A joint consensus statement from the European Atherosclerosis Society and European Federat. Clin Chem. 2016;62(7):930-46.

4. Langsted A, Freiberg JJ NB. Fasting and non-fasting lipid levels: influence of normal food intake on lipids, lipoproteins, apolipoproteins, and cardiovascular risk prediction. Circulation. 2008;118:2047-2056.

5. Langsted A, Freiberg JJ, Nordestgaard BG. Fasting and nonfasting lipid levels influence of normal food intake on lipids, lipoproteins, apolipoproteins, and cardiovascular risk prediction. Circulation. 2008;118(20):2047-56. 
6. Nordestgaard BG, Benn M, Schnohr P T-HA. Nonfasting triglycerides and and risk of myocardial infarction, ischemic heart disease, and death in men and women. JAMA. 2007;298:299-308.

7. Devaraj S, Cao J, Roper SM. To fast or not to fast?: Comments on the consensus statement from the European atherosclerosis society/European federation of clinical chemistry and laboratory medicine. Arch Pathol Lab Med. 2017;141(4):487-9.

8. Bansal S, Buring JE, Rifai N, Mora S, Sacks FM RP. Fasting compared with nonfasting triglycerides and risk of cardiovascular events in women. JAMA. 2007;298:309-16.

9. Mora S, Rifai N, Burring JE RP. Fasting compared with non fasting lipids and Apolipoproteins for predicting incident cardiovascular events. Circulation. 2008;118:993-1001.

10. McKenney JM, Davidson MH, Jacobson TA GJ. Final conclusions of the National Lipid Association statin safety assessment task force. Am J Cardiol. 2006;97:89C-95C.

11. Nordestgaard BG, initiative for the EAS (EAS) and the EF of CC and LM (EFLM) joint consensus, Langsted A, initiative for the EAS (EAS) and the EF of CC and LM (EFLM) joint consensus, Mora S, initiative for the EAS (EAS) and the EF of CC and LM (EFLM) joint consensus, et al. Fasting is not routinely required for determination of a lipid profile: clinical and laboratory implications including flagging at desirable concentration cut-points-a joint consensus statement from the European Atherosclerosis Society and European Federa. Eur Heart J [Internet]. 2016;37(25):1944 58. Available from: http://dx.doi.org/10.1093/eurheartj/ehw152

12. Nauck M, Warnick GR RN. Methods for measurement of LDL-cholesterol: a critical assessment of direct measurement by homogeneous assays versus calculation. Clin Chem. 2002;48(2):236-54.

13. Stone NJ, Robinson JG, Lichtenstein AH et al. ACC/AHA guideline on the treatment of blood cholesterol to reduce atherosclerotic cardiovascular risk in adults: a report of the American College of Cardiology/American Heart Association Task Force on Practice guidelines. J Am Coll Cardiol. 2014;63:2889-2934.

14. Rahman F, Blumenthal RS, Jones SR, Martin SS, Gluckman TJ, Whelton SP. Fasting or Non-fasting Lipids for Atherosclerotic Cardiovascular Disease Risk Assessment and Treatment? Curr Atheroscler Rep [Internet]. $2018 ; 20$ (3) (14):4-9. Available from:

http://ovidsp.ovid.com/ovidweb.cgi?T $=J S \& C S C=Y \& N E W S=N \& P A G E=$ fulltext $\& D=$ emexb\&AN $=620751414$

http:/ / oxfordsfx.hosted.exlibrisgroup.com/oxford?sid=OVID:embase\&id=pmid:\&id=doi:10.1007\%2Fs11883-018-

0713-2\&issn $=1523-3804 \&$ isbn $=\&$ volume $=20 \&$ issue $=3 \&$ spage $=14 \&$ pages $=\&$ dat

15. Mansbach CM, Siddiqi SA. The biogenesis of chylomicrons. Annu Rev Physiol. 2010;72(1):315-33.

16. Sniderman A, Vu H CK. Effect of moderate hypertriglyceridemia on the relation of plasma total and LDL apo B levels. Atherosclerosis. 1991;89(2-3):109-16.

17. Friedewald WT, Levy RI FD. Estimation of the concentration of low-density lipoprotein cholesterol in plasma, without use of the preparative ultracentrifuge. Clin Chem. 1972;18:499-502.

18. Langsted A, Nordestgaard BG. Nonfasting lipid profiles: The way of the future. Clin Chem. 2015;61(9):1123-5.

19. Friedewald WT, Levy RI FD. Estimation of the concentration of low- density lipoprotein cholesterol in plasma, without use of the preparative ultracentrifuge. Clin Chem. 1972;18:499-502.

20. Cartier LJ, Collins C, Lagacé M, Douville P. Comparison of fasting and non-fasting lipid profiles in a large cohort of patients presenting at a community hospital. Clin Biochem [Internet]. 2018;52:61-6. Available from: http://dx.doi.org/10.1016/j.clinbiochem.2017.11.007

21. Varbo A, Benn M, Tybjaerg-Hansen A, Jorgensen AB, Frikke-Schmidt R NB. Remnant cholesterol as a causal risk factor for ischemic heart disease. J Am Coll Cardiol. 2013;61:427-436.

22. Simundic AM, Cornes M, Grankvist K, Lippi G NM. Standardization of collection requirements for fasting samples: for the Working Group on Preanalytical Phase (WG-PA) of the European Federation of Clinical Chemistry and Laboratory Medicine (EFLM). Clin Chim Acta. 2014;432:33-37.

23. JM G. Should we fast before we measure our lipids? Arch Intern Med. 2012;172:1705-1706.

24. Farukhi Z, Mora S. Re-assessing the role of non-fasting lipids; a change in perspective. Ann Transl Med [Internet]. 2016;4(21):431-431. Available from: http://atm.amegroups.com/article/view/12394/12864

25. Pati SS, Singh AK. Can non-fasting and fasting lipid profile be mutually exclusive? An Indian perspective. J Investig Med. 2017;65(7):e3. 
26. Nigam PK. Serum lipid profile: Fasting or non-fasting? Indian J Clin Biochem. 2011;26(1):96-7.

27. Yang D, Cai Q, Qi X, Zhou Y, Ph D. Postprandial Lipid Concentrations and Daytime Biological Variation of Lipids in a Healthy Chinese Population. 2018;431-9.

28. (UK) NCGC. Lipid Modification: Cardiovascular Risk Assessment and the Modification of Blood Lipids for the Primary and Secondary Prevention of Cardiovascular Disease. London. Natl Inst Heal Care Excell. 2014;

29. Post P, Distribute OR, Widely AS, Appropriate AS. Lipids - Fasting versus Non-Fasting - Clinical Considerations and Laboratory Specimen Collection Policy for Community Patients (Update from 2014 Bulletin). 2017;(1):1-3.

30. Umakanth M, Ibrahim M. Fasting and Non-Fasting Lipid Profile among Healthcare Workers at Teaching Hospital Batticaloa Sri Lanka. J Biosci Med [Internet]. 2018;6(7):15-22. Available from: http://www.scirp.org/journal/doi.aspx?DOI=10.4236/jbm.2018.67002 